\title{
Rigorous Criterion for Reentrance in the Spin-1/2 Ising-Heisenberg Model on Diamond-Like Decorated Bethe Lattices
}

\author{
J. STREČKA ${ }^{a}$ AND C. EKIZ ${ }^{b}$ \\ ${ }^{a}$ Department of Theoretical Physics and Astrophysics, Faculty of Science, P.J. Šafárik University \\ Park Angelinum 9, 04001 Košice, Slovak Republic \\ ${ }^{b}$ Department of Physics, Faculty of Science, Adnan Menderes University \\ 09010 Aydin, Turkey
}

\begin{abstract}
The spin-1/2 Ising-Heisenberg model on diamond-like decorated Bethe lattices is exactly solved with the help of decoration-iteration transformation and exact recursion relations. It is shown that the model under investigation exhibits reentrant phase transitions whenever a sufficiently high coordination number of the underlying Bethe lattice is considered.
\end{abstract}

PACS numbers: 05.50.+q, 75.10.-b, 75.10.Kt, 75.40.-s

\section{Introduction}

The ferromagnetic spin-1/2 Ising-Heisenberg model on planar lattices currently attracts a great deal of attention as it may exhibit a variety of interesting phenomena such as a quantum critical point [1,2], peculiar spontaneous antiferromagnetic long-range order [1] or several unusual spin-liquid ground states [2]. In this work, the spin- $1 / 2$ Ising-Heisenberg model on diamond-like decorated Bethe lattices will be treated exactly with the aim to study its ground-state and finite-temperature phase diagrams.

\section{Ising-Heisenberg model and its exact solution}

Consider the spin- $1 / 2$ Ising-Heisenberg model on the diamond-like decorated Bethe lattices schematically illustrated on the left hand side of Fig. 1. In this figure, the full circles denote lattice positions of the Ising spins $\mu$, the empty circles label lattice positions of the Heisenberg spins $S$ and the parameter $q$ is the coordination number of the underlying Bethe lattice. For further convenience, the total Hamiltonian can be defined as a sum of the bond Hamiltonians, i.e. $\hat{\mathcal{H}}=\sum_{k} \hat{\mathcal{H}}_{k}$, where each bond Hamiltonian $\hat{\mathcal{H}}_{k}$ involves all the interaction terms belonging to the $k$-th diamond unit, enclosed with an ellipse in Fig. 1 (see Fig. 1)

$$
\begin{gathered}
\hat{\mathcal{H}}_{k}=-J\left[\Delta\left(\hat{S}_{k 1}^{x} \hat{S}_{k 2}^{x}+\hat{S}_{k 1}^{y} \hat{S}_{k 2}^{y}\right)+\hat{S}_{k 1}^{z} \hat{S}_{k 2}^{z}\right] \\
-J_{\mathrm{I}}\left(\hat{S}_{k 1}^{z}+\hat{S}_{k 2}^{z}\right)\left(\hat{\mu}_{k 1}^{z}+\hat{\mu}_{k 2}^{z}\right) .
\end{gathered}
$$

In above, $\hat{S}_{i}^{\alpha}(\alpha=x, y, z)$ and $\hat{\mu}_{j}^{z}$ label spatial components of the spin- $1 / 2$ operator, the former term $J$ accounts for the $X X Z$ interaction between the nearest-neighbour Heisenberg spins, $\Delta$ is a spatial anisotropy in this interaction and the latter term $J_{\mathrm{I}}$ accounts for the Ising-type interaction between the nearest-neighbour Ising and Heisenberg spins, respectively.

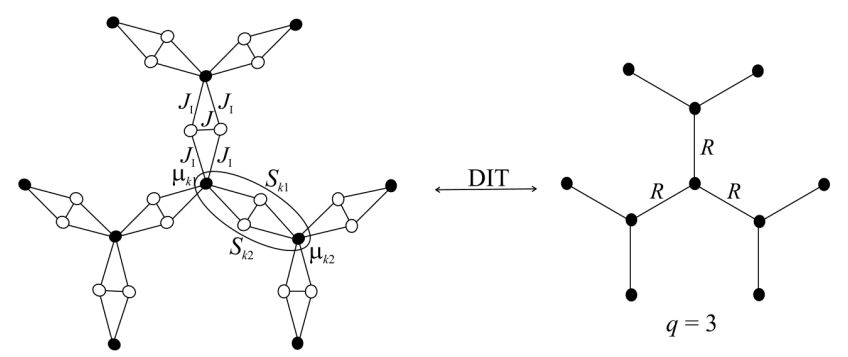

Fig. 1. A schematic representation of the decorationiteration transformation (DIT) between the spin- $1 / 2$ Ising-Heisenberg model on the diamond-like decorated Bethe lattice (left) and the spin- $1 / 2$ Ising model on the simple Bethe lattice with the coordination number $q=3$ (right). The full (empty) circles denote lattice positions of the Ising (Heisenberg) spins and the ellipse demarcates all interaction terms belonging to the $k$-th bond Hamiltonian (1).

A crucial step of our approach lies in evaluation of the partition function. Due to a commutability of different bond Hamiltonians, the partition function can be partially factorized to a product of bond partition functions

$$
\mathcal{Z}_{\mathrm{IHM}}=\sum_{\left\{\mu_{i}\right\}} \prod_{k=1}^{N q / 2} \operatorname{Tr}_{k} \exp \left(-\beta \hat{\mathcal{H}}_{k}\right)=\sum_{\left\{\mu_{i}\right\}} \prod_{k=1}^{N q / 2} \mathcal{Z}_{k}
$$

where $\beta=1 /\left(k_{\mathrm{B}} T\right), k_{\mathrm{B}}$ is Boltzmann's constant, $T$ denotes absolute temperature, $N$ is the total number of the Ising spins, the symbol $\operatorname{Tr}_{k}$ stands for a trace over degrees 
of freedom of the $k$-th Heisenberg spin pair and the summation $\sum_{\left\{\mu_{i}\right\}}$ runs over all available configurations of the Ising spins. A calculation of the bond partition function $\mathcal{Z}_{k}$ can easily be accomplished through a direct diagonalization of the bond Hamiltonian (1). Subsequently, the gained expression for $\mathcal{Z}_{k}$ will depend just on two outer Ising spins and can be replaced with the use of generalized decoration-iteration transformation [2]:

$$
\begin{aligned}
& \mathcal{Z}_{k}\left(\mu_{k 1}^{z}, \mu_{k 2}^{z}\right)=2 \exp (\beta J / 4) \cosh \left[\beta J_{\mathrm{I}}\left(\mu_{k 1}^{z}+\mu_{k 2}^{z}\right)\right] \\
& \quad+2 \exp (-\beta J / 4) \cosh (\beta J \Delta / 2) \\
& \quad=A \exp \left(\beta R \mu_{k 1}^{z} \mu_{k 2}^{z}\right) .
\end{aligned}
$$

The mapping parameters $A$ and $R$ can directly be obtained from the decoration-iteration transformation (3), which holds quite generally if and only if

$$
\begin{aligned}
& A=\left[\mathcal{Z}_{k}(1 / 2,1 / 2) \mathcal{Z}_{k}(1 / 2,-1 / 2)\right]^{1 / 2}, \\
& \beta R=2 \ln \left[\mathcal{Z}_{k}(1 / 2,1 / 2)\right]-2 \ln \left[\mathcal{Z}_{k}(1 / 2,-1 / 2)\right] .
\end{aligned}
$$

Substituting the algebraic transformation (3) into the formula (2) one gets a precise mapping relationship between partition functions of the spin- $1 / 2$ Ising-Heisenberg model on the diamond-like decorated Bethe lattice and the corresponding spin- $1 / 2$ Ising model on the simple Bethe lattice (see Fig. 1)

$$
\mathcal{Z}_{\mathrm{IHM}}\left(\beta, J, \Delta, J_{\mathrm{I}}\right)=A^{N q / 2} \mathcal{Z}_{\mathrm{IM}}(\beta, R) .
$$

Let us note that the partition function of the spin- $1 / 2$ Ising model on the Bethe lattice can exactly be calculated by making use of the rigorous recursion relations [3] and hence, our exact calculation is formally completed. Using the mapping equivalence (6) between both partition functions, the sublattice magnetization of the Ising spins directly equals to the single-site magnetization of the corresponding spin-1/2 Ising model on the simple Bethe lattice

$$
m_{i}^{z} \equiv\left\langle\hat{\mu}_{k}^{z}\right\rangle_{\mathrm{IHM}}=\left\langle\hat{\mu}_{k}^{z}\right\rangle_{\mathrm{IM}} \equiv m_{\mathrm{IM}}(\beta R) .
$$

Above, the symbols $\langle\ldots\rangle_{\mathrm{IHM}}$ and $\langle\ldots\rangle_{\mathrm{IM}}$ mark canonical ensemble average performed within two models connected through the mapping relation (6). Let us note furthermore that the corresponding exact result for the magnetization $m_{\mathrm{IM}}$ can be also found with the help of recursion relations [3]. The sublattice magnetization of the Heisenberg spins follows from the formula:

$$
\begin{aligned}
m_{h}^{z} & \equiv\left\langle\hat{S}_{k}^{z}\right\rangle_{\mathrm{IHM}} \\
& =\frac{m_{\mathrm{IM}} \sinh \left(\beta J_{\mathrm{I}}\right)}{\cosh \left(\beta J_{\mathrm{I}}\right)+\exp \left(-\frac{\beta J}{2}\right) \cosh \left(\frac{\beta J \Delta}{2}\right)} .
\end{aligned}
$$

\section{Results and discussion}

First, let us take a closer look at the ground state of the spin-1/2 Ising-Heisenberg model with both ferromagnetic interactions $\left(J>0, J_{\mathrm{I}}>0\right)$. The classical ferromagnetic phase with all Ising as well as Heisenberg spins aligned parallel constitutes the ground state whenever the exchange anisotropy $\Delta<\Delta_{\mathrm{c}}=1+2 J_{\mathrm{I}} / J$. In the opposite case $\Delta>\Delta_{\mathrm{c}}$, the remarkable spin-liquid phase constitutes the ground state and this disordered phase can be characterized through a complete randomness of the Ising spins and the quantum entanglement of the Heisenberg spin pairs thoroughly described in Ref. [2]. An origin of this unusual disordered phase lies in the geometric frustration, which is triggered by a competition between the easy-axis Ising interaction and the easy-plane $X X Z$ Heisenberg interaction [2].

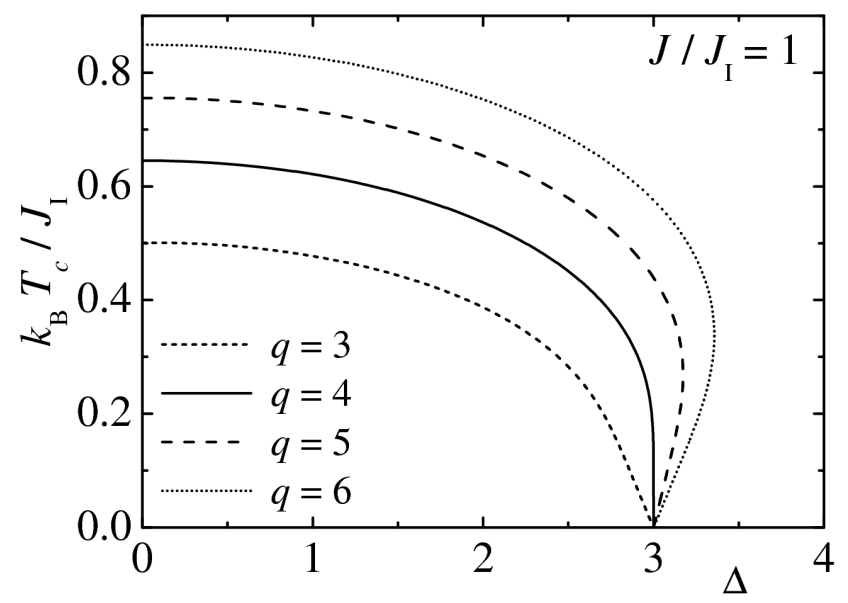

Fig. 2. Critical temperature as a function of the exchange anisotropy $\Delta$ for one selected ratio $J / J_{\mathrm{I}}=1$ and several values of the coordination number $q$.

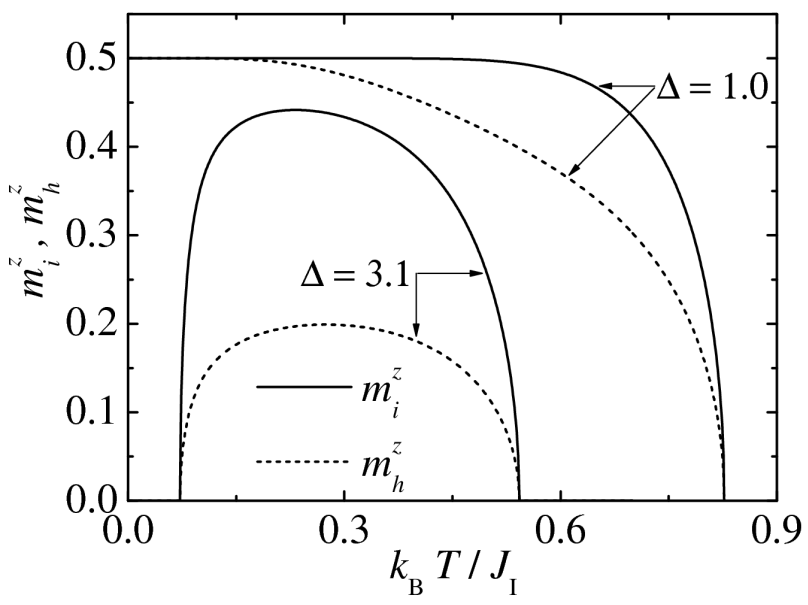

Fig. 3. Thermal variations of both spontaneous sublattice magnetizations for $J / J_{\mathrm{I}}=1, q=6$ and two different values of the exchange anisotropy $\Delta$.

Figure 2 shows the critical temperature as a function of the exchange anisotropy $\Delta$ for one particular ratio $J / J_{\mathrm{I}}=1$ and several values of the coordination number $q$. As one can see, the critical temperature vs. exchange anisotropy dependence basically changes with the coordination number $q$ of the underlying Bethe lattice. The critical lines are approaching the zero-temperature phase boundary between the classical ferromagnetic phase and 
disordered phase at $\Delta_{\mathrm{c}}$ with a negative (positive) slope for the diamond-like decorated Bethe lattices with the coordination number $q<4(q>4)$ and with an infinite gradient for the particular case with the coordination number $q=4$. Owing to this fact, reentrant phase transitions can be observed for $\Delta \gtrsim \Delta_{\mathrm{c}}$ only when assuming the diamond-like decorated Bethe lattices with a sufficiently high coordination number $q>4$. Thermal variations of both sublattice magnetizations, which are depicted in Fig. 3, provide an independent confirmation of the aforedescribed reentrance.

In conclusion, it is worthy to note that the rigorous procedure developed on the grounds of decoration-iteration transformation and exact recursion relations can be readily adapted to treat the investigated model in a presence of external magnetic field, which will be examined in detail in our forthcoming work.

\section{Acknowledgments}

This work was supported under the grants VEGA 1/0431/10 and VVGS 2/09-10.

\section{References}

[1] J. Strečka, M. Jaščur, Phys. Rev. B 66, 174415 (2002).

[2] J. Strečka, M. Jaščur, Acta Phys. Slov. 56, 65 (2006).

[3] R.J. Baxter, Exactly Solved Models in Statistical Mechanics, Academic, New York 1982. 